\title{
APRESENTAÇÃO DO DOSSIÊ "EDUCAÇÃO PROFISSIONAL TECNOLÓGICA"
}

\author{
PRESENTATION OF THE DOSSIER \\ "TECHNOLOGICAL PROFESSIONAL EDUCATION" \\ PRESENTACIÓN DEL DOSSIER \\ "EDUCACIÓN PROFESIONAL TECNOLÓGICA "
}

Célia Regina Otranto

(UFRRJ, Brasil)

\section{do) https://doi.org/10.29404/rtps-v5i8.414}

RESUMO: 0 texto apresenta o Dossiê "Educação Profissional Tecnológica" organizado pela Dra Célia Regina Otranto, da Universidade Federal Rural do Rio de Janeiro (UFRRJ). Na apresentação, a organizadora apresenta a estruturação do dossiê, os autores e as ideias centrais de cada um dos artigos componentes.

Palavras-chave: Ensino Superior, Educação Profissional Tecnológica, Política Educacional.

\begin{abstract}
The text presents the Dossier "Technological Professional Education" organized by $\mathrm{Dr}^{\mathrm{a}}$ Celia Regina Otranto, from the Universidade Federal Rural do Rio de Janeiro (UFRRJ). In this presentation, the organizer presents the structure of the dossier, the authors, and the central ideas of the component articles.
\end{abstract}

Keywords: Higher Education, Technological Professional Education, Educational Policy.
RESUMEN: El texto presenta el Dossier "Educación Profesional Tecnológica" organizado por la Dra. Celia Regina Otranto, de la Universidade Federal Rural do Rio de Janeiro (UFRRJ). En esta presentación, la organizadora presenta la estructura del dossier, los autores y las ideas centrales de los artículos componentes.

Palabras clave: Educación Superior, Educación Profesional Tecnológica, Política Educativa.

É com muita satisfação que apresento neste espaço o Dossiê "Educação Profissional Tecnológica", que agrega 18 autores de 15 diferentes instituições. Este número da RTPS Revista Trabalho Política e Sociedade traz 10 artigos sobre a educação superior profissional redigidos por pesquisadores de diversas regiões do país que vêm se debruçando sobre a temática. Congrega, portanto, textos que podem vir a subsidiar estudos futuros sobre o assunto, enriquecendo-os sobremaneira com a leitura dos artigos aqui disponibilizados, tendo em vista que apresentam diferentes perspectivas de análise da educação superior profissional, com a possibilidade real de contribuir nas mais diversas investigações que envolvem o espectro da temática. 
A organização deste Dossiê seguiu duas diretrizes: partir dos temas mais gerais para os mais específicos e manter a proximidade de temas semelhantes. Com isso, pretendemos seguir uma lógica, que vem sendo adotada por muitos estudiosos da área de Ciências Humanas, de localizar o problema de pesquisa dentro de um espectro mais amplo, para facilitar sua contextualização, para depois analisá-lo em um espaço circunscrito ao tema principal. Consideramos aqui como espectros mais amplos os três primeiros temas: financiamento, avaliação institucional e expansão. Os temas mais específicos foram agrupados em dois eixos: a) os cursos tecnológicos, com três artigos; e b) a formação para o trabalho e a formação para o trabalho docente, representado por quatro artigos. Porém, esta divisão foi adotada somente para favorecer uma organização lógica, ela não é estanque e um tema não exclui o outro. Pelo contrário, os temas gerais integram a fundamentação teórica dos temas específicos e os específicos acabam se entrelaçando de tal forma que, ao final da leitura do Dossiê o leitor tem uma ideia bem mais abrangente da temática.

Iniciamos, então, com a apresentação dos temas considerados mais gerais: financiamento, avaliação institucional e expansão da educação superior profissional.

O financiamento ganha ênfase no artigo intitulado Expansão da Rede Federal de Educação Profissional e os desafios do financiamento (2013-2018), redigido por João Ribeiro dos Santos Filho, do Tribunal de Contas da União (TCU), e Vera Lúcia Jacob Chaves, da Universidade Federal do Pará (UFPA). O artigo discute a expansão da Rede Federal de Educação Profissional, com foco nos Institutos Federais de Educação, Ciência e Tecnologia (IFs) e Centros Federais de Educação Tecnológica (CEFETs), analisando os impactos da redução dos recursos financeiros no funcionamento dessas instituições. Merece destaque a riqueza de dados estatísticos amparada em relatórios de execução orçamentária do governo federal e de dados do Censo da Educação Superior. O artigo analisa a queda do financiamento das instituições de educação superior, após a aprovação da Emenda Constitucional n 95/2016 (BRASIL, 2016), alertando para a possibilidade de agravamento do problema a curto e médio prazo (SANTOS FILHO; CHAVES, 2020).

Liz Denize de Carvalho Paiva e Nádia Maria Pereira de Souza, ambas da Universidade Federal Rural do Rio de Janeiro (UFRRJ), redigiram o texto sobre avaliação Institucional que tem por título $A$ avaliação institucional no Instituto Federal de Educação, Ciência $e$ Tecnologia a partir dos eixos dos SINAES. O artigo analisa as principais mudanças no Instrumento de Avaliação conhecido como SINAES e suas repercussões nos IFs, levando em consideração a atualização ocorrida no ano de 2017, ocasionada pela Portaria $n^{\circ}$ 1.382/2017 (BRASIL, 2017). Destaca os principais paradigmas presentes na avaliação institucional das Instituições de Educação Superior, apontando as principais diferenças entre os IFs e as universidades federais, que podem interferir nos resultados dessa avaliação (PAIVA; SOUZA, 2020). 
A temática da expansão da educação superior foi explorada no artigo Expansão da Educação Superior na Rede Federal de Educação Profissional, Científica e Tecnológica (2008-2018) redigido por Júlia Milena da Paixão Oliveira e André Rodrigues Guimarães, ambos da Universidade Federal do Amapá (UNIFAP). As análises de dez anos dessa expansão estão apoiadas em pesquisa documental e em dados do Censo da Educação Superior do Instituto Nacional de Estudos e Pesquisas Educacionais Anísio Teixeira (INEP). Fundamentados no materialismo histórico e dialético, os autores apontam a relação histórica da educação superior com a educação profissional e a expansão da educação superior advinda da criação da Rede Federal de Educação Profissional, Científica e Tecnológica, pela Lei $n^{\circ}$ 11.892/2008 (BRASIL, 2008). Destacam a significativa contribuição das Instituições que integram essa Rede na expansão dos cursos de graduação no Brasil (OLIVEIRA; GUIMARÃES, 2020).

Na sequência, são apresentados três artigos que têm como foco principal de estudo: educação tecnológica/cursos superiores de tecnologia.

Zuleide S. Silveira, da Universidade Federal Fluminense (UFF), é a autora do texto intitulado Concepção burguesa de educação tecnológica, desenvolvimento econômico e desenvolvimento científico-tecnológico. $\mathrm{O}$ artigo abrange estudos desenvolvidos em um período de 30 anos (1960-1990), analisando o desenvolvimento da concepção burguesa de educação tecnológica no bojo do processo de institucionalização e implementação da política de Ciência e Tecnologia. O texto está organizado em três partes: a) pensamento econômico e educação, onde reexamina a teoria do capital humano; b) a subsunção da política educacional à Política De Ciência e Tecnologia; c) a concepção e abrangência da educação tecnológica na perspectiva burguesa. Discute o lugar das Ciências Humanas e Sociais Aplicadas no quadro educacional brasileiro, para, ao final, esclarecer o que chama de educação tecnológica burguesa (SILVEIRA, 2020).

Retrato da Expansão e avaliação dos Cursos Superiores de Tecnologia é o título do artigo elaborado por José Vieira de Sousa, da Universidade de Brasília (UnB). O autor associa a expansão dos Cursos Superiores de Tecnologia (CSTs) com os problemas relacionados à avaliação dos cursos e das instituições que os oferecem, apoiando-se em análises das principais tendências, controvérsias e motivação inerentes ao processo. $O$ texto está estruturado em quatro partes: a) os CSTs no contexto da Reforma da Educação profissional e tecnológica; b) contextualização dos CSTs na expansão da educação superior brasileira; c) a expansão dos CSTs no Brasil; d) característica dos processos de avaliação e regulação dos CSTs. A organização dada pelo autor permite acompanhar cada passo da pesquisa, proporcionando ao leitor uma visão ampla do assunto, não somente no tempo, como também nos diferentes espaços institucionais e modalidades, quer oferecidas de forma presencial ou a distância (SOUSA, 2020). 
Celia Veiga, da Escola de Inteligência de Segurança Pública do Estado do Rio de Janeiro (ESISPERJ) e Bruno de Oliveira Figueiredo, da Fundação de Apoio à Escola Técnica (FAETEC), redigiram o artigo intitulado A proposição do Curso Superior de Tecnologia para formação do trabalhador de novo tipo. Os autores desenvolvem análises a respeito do papel dos Cursos Superiores de Tecnologia na formação para o trabalho, tomando como referência o contexto da crise orgânica do capital e a consequente recomposição burguesa, constantemente em busca da manutenção da sua hegemonia. Examinam o reordenamento mundial do trabalho educacional e a ressignificação e reestruturação da educação superior como "educação terciária", tendo como foco a educação profissional tecnológica. O texto está organizado em três partes: a) recomposição burguesa e pedagogia política do capital; b) a atuação dos organismos internacionais na proposição dos CSTs; c) o CST e a formação do trabalhador de novo tipo. Apesar de ter sido situado no eixo Cursos Tecnológicos/Cursos Superiores de Tecnologia, é possível identificar que os autores transitam também pelo eixo da formação para o trabalho, corroborando a afirmativa inserida no início dessa apresentação de que os textos se integram, se complementam, a fim de garantir ao leitor uma visão mais abrangente do tema (VEIGA; FIGUEIREDO, 2020).

Passamos em seguida à apresentação dos quatro textos finais, que abordam pesquisas voltadas para o tema: formação para o trabalho/formação para o trabalho docente.

O assunto é discutido, primeiramente, por Priscila Caetano Betim, do Instituto Federal de Educação, Ciência e Tecnologia do Rio de Janeiro (IFRJ), e Deise Mancebo, da Universidade do Estado do Rio de Janeiro (UERJ), no artigo "Novos Caminhos" e formação para o trabalho nos Institutos Federais, que analisa as políticas públicas mais atuais (20192020). O texto se inicia apresentando o cenário educacional no ano de 2018, quando foi eleito o atual presidente da república, Jair Bolsonaro, para em seguida abordar as mudanças ocasionadas pelas políticas públicas no campo da educação, adotadas a partir do ano de 2019. O ponto central da análise é formação para o trabalho nos IFs, e as autoras elegeram o Programa intitulado Novos Caminhos (BRASIL, 2019), para investigar a concepção de formação para o trabalho que permeia o documento e, consequentemente, a política pública do atual governo e seus possíveis impactos no cenário educacional brasileiro (BETIM; MANCEBO, 2020).

Anderson Teixeira Boanafina e Mônica Werner Wermelinger, ambos da Fundação Oswaldo Cruz (FIOCRUZ), apresentaram o texto intitulado $A$ formação docente nos institutos federais e a educação profissional em saúde: um debate necessário. No artigo, os autores discutem o tema da falta de cursos específicos para formar professores para a educação profissional, tendo como foco os cursos voltados para a área de saúde, especialmente os técnicos de enfermagem. Denunciam que, apesar da ampliação crescente das demandas por trabalhadores, indispensável ao Sistema Único de Saúde (SUS), a formação de técnicos e de licenciados em enfermagem têm sido subdimensionadas pela 
educação pública em geral e pelos IFs, em particular. Defendem que a expansão da educação profissional depende, essencialmente, da formação de docentes que conduzam a educação profissional pública sob uma nova identidade, que vá além da integração com o ensino médio, e que seja centrada no ser humano e não no capital. No texto consta o alerta de que a saúde precisa de profissionais plenamente qualificados para atender às necessidades do SUS, conforme está sendo comprovado, na atualidade, pela pandemia ocasionada pelo vírus COVID-19 (BOANAFINA; WERMELINGER, 2020).

Licenciaturas na Rede Federal Profissional e Tecnológica: um estudo com dados dos concluintes, é o título do artigo redigido por Maria Flávia Batista Lima, da Universidade Federal do ABC (UFABC), e Gladys Beatriz Barreyro, da Universidade de São Paulo (USP). O texto aborda, primeiramente, a expansão da Rede Federal de Educação Profissional e Tecnológica, de forma geral, para em seguida tratar da expansão das licenciaturas na Rede, tomando como referência os dados estatísticos do Censo da Educação Superior. Aponta o crescimento desses cursos, oferecidos de forma presencial ou por meio de educação a distância, no período 2009-2018, tendo como foco principal de análise os concluintes das licenciaturas nas áreas de: Matemática, Física, Química e Biologia. Discute a formação de professores em um espaço que, anteriormente, era direcionado aos cursos médio e técnico e compara os dados obtidos na Rede Federal de Educação Profissional e Tecnológica com os dados das demais instituições públicas e privadas da educação superior brasileira (LIMA; BARREYRO, 2020).

O último texto deste Dossiê foi apresentado por Tatiana das Mercês, da Secretaria de Estado de Educação do Espírito Santo (SEEduc/ES), e Marcelo Lima, da Universidade Federal do Espírito Santo (UFES), e tem por título Especificidade da docência no Instituto Federal de Educação, Ciência e Tecnologia do Espírito Santo. O artigo se inicia com reflexões a respeito do trabalho docente apontando suas especificidades, visto pelos autores como um instrumento de luta política. Passa, em seguida, para as análises referentes às características dos Institutos Federais e a verticalização do ensino para, na sequência, apresentar o Instituto Federal de Educação, Ciência e Tecnologia do Espírito Santo (IFES). No IFES a pesquisa tem como foco alunos e professores do curso técnico em mecânica. Em relação aos alunos, investiga o perfil e, no que diz respeito aos professores, aponta as especificidades da docência em um mesmo curso de mecânica, oferecido de forma integrada ou concomitante ao ensino médio (MERCÊS; LIMA, 2020).

A apresentação dos diferentes artigos teve por objetivo despertar a curiosidade do leitor para a leitura integral dos textos. Nesse sentido, propositadamente, não divulgamos as conclusões dos estudos, para que cada leitor busque essa informação no corpo dos artigos, consultando, inclusive, as referências bibliográficas que direcionam as reflexões e análises em cada um dos textos. Foi a forma que encontramos de incentivar futuros estudos 
e de prestigiar os pesquisadores que se dedicam a estudar um tema ainda pouco explorado: a educação profissional tecnológica.

\section{Referências}

BETIM, Priscila Caetano; MANCEBO, Deise. 'Novos Caminhos' e a formação para o trabalho nos Institutos Federais". RTPS - Rev. Trabalho, Política e Sociedade, Vol. V, n 8, p. X-Y, jan-jun/2020.

BOANAFINA. Anderson T.; WERMELINGER, Mônica. A formação docente nos institutos federais e a educação profissional em saúde: um debate necessário. RTPS - Rev. Trabalho, Política e Sociedade, Vol. V, n 8, p. X-Y, jan-jun/2020.

BRASIL. Emenda Constitucional $\mathbf{n}^{\circ}$ 95, de 15 de dezembro de 2016. Altera o Ato das Disposições Constitucionais Transitórias, para instituir o Novo Regime Fiscal, e dá outras providências. Brasília, DF, 2016. Disponível em: http://www.planalto.gov.br/ccivil 03/constituicao/Emendas/Emc/emc95.ht m Acesso em: 20/02/2020.

BRASIL. Portaria $n^{\circ} 1.382$, de 31 de outubro de 2017. Aprova, em extratos, os indicadores dos Instrumentos de Avaliação Institucional Externa para os atos de credenciamento, recredenciamento e transformação de organização acadêmica nas modalidades presencial e a distância do Sistema Nacional de Avaliação da Educação Superior - SINAES. Diário Oficial [da] República Federativa do Brasil, de 01/11/2017 nº 210, Seção 1, p. 14.

BRASIL. Congresso Nacional. Lei $n^{\circ} 11.892$, de 29 de dezembro de 2008. Institui a Rede Federal de Educação Profissional Científica e Tecnológica e dá outras providências. Diário Oficial [da] República Federativa do Brasil, Brasília, DF, Seção 1, p. 1, 30 dez. 2008.

BRASIL. Um novo modelo em Educação Profissional e Tecnológica - Concepção e Diretrizes. Brasília: Ministério da Educação. Secretária de Educação Profissional e Tecnológica, $2010 . \quad 43 \quad$ p. $\quad$ Disponível em http://portal.mec.gov.br/index.php?option=com_docman\&view=download\&alias=6691if-concepcaoediretrizes\&category_slug=setembro-2010-pdf\&Itemid=30192. Acesso em 25 de jan. 2020.

FIGUEIREDO, Bruno de Oliveira; VEIGA, Celia. A proposição do Curso Superior de Tecnologia para formação do trabalhador de novo tipo. RTPS - Rev. Trabalho, Política e Sociedade, Vol. V, no 8, p. X-Y, jan-jun/2020.

LIMA, Maria Flávia Batista; BARREYRO, Gladys Beatriz Licenciaturas na Rede Federal Profissional e Tecnológica: um estudo com dados dos concluintes. RTPS - Rev. Trabalho, Política e Sociedade, Vol. V, no 8, p. X-Y, jan-jun/2020.

MERCÊS, Tatiana das; LIMA, Marcelo. Especificidade da docência no Instituto Federal de Educação, Ciência e Tecnologia do Espírito Santo. RTPS - Rev. Trabalho, Política e Sociedade, Vol. V, nº 8, p. X-Y, jan-jun/2020. 
OLIVEIRA, Júlia Milena P; GUIMARÃES, André Rodrigues. Expansão da Educação Superior na Rede Federal de Educação Profissional, Científica e Tecnológica (2008-2018). RTPS Rev. Trabalho, Política e Sociedade, Vol. V, nº 8, p. X-Y, jan-jun/2020.

PAIVA, Liz Denize Carvalho; SOUZA Nádia Maria Pereira de. A avaliação institucional no Instituto Federal de Educação, Ciência e Tecnologia a partir dos eixos dos SINAES. RTPS Rev. Trabalho, Política e Sociedade, Vol. V, no 8, p. X-Y, jan-jun/2020.

SANTOS FILHO, João Ribeiro dos; CHAVES, Vera Lúcia Jacob. Expansão da Rede Federal de Educação Profissional e os desafios do financiamento (2013-2018). RTPS - Rev. Trabalho, Política e Sociedade, Vol. V, nº 8, p. X-Y, jan-jun/2020.

SILVEIRA, Zuleide S. Concepção burguesa de educação tecnológica, desenvolvimento econômico e desenvolvimento científico-tecnológico. RTPS - Rev. Trabalho, Política e Sociedade, Vol. V, no 8, p. X-Y, jan-jun/2020.

SOUZA, José Vieira de. Retrato da Expansão e avaliação dos Cursos Superiores de Tecnologia. RTPS - Rev. Trabalho, Política e Sociedade, Vol. V, nº 8, p. X-Y, jan-jun/2020.

\section{Informações sobre a autora:}

\section{Celia Regina Otranto}

Doutora em Ciências Sociais pela Universidade Federal Rural do Rio de Janeiro (UFRRJ), com Pós-Doutorado em Educação pela Universidade Estadual de Campinas (UNICAMP). Aposentou-se como professora titular da UFRRJ, onde até hoje integra o quadro docente do Programa de Educação, Contextos Contemporâneos e Demandas Populares (PPGEduc), do qual foi Coordenadora de 2012 a 2014 e Vice-Coordenadora de 2014 a 2016 e de 2010 a 2012. É líder do Grupo de Estudos e Pesquisas da História da Universidade Rural (GEPHUR), é pesquisadora da Rede UNIVERSITAS-BR, ligada ao GT-11 da Associação Nacional de Pesquisa e Pós-Graduação em Educação (ANPEd), do qual é atual Coordenadora.

ORCID iD: https://orcid.org/0000-0001-8914-5705

E-mail: celiaotranto@gmail.com

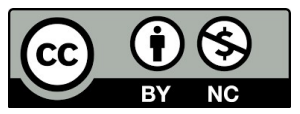

Esta obra está licenciada com uma Licença 